\title{
Structural Analysis of Metal Interactions with the Dinucleotide Duplex, dCG·dCG, Using Ion Mobility Mass Spectrometry
}

Erin Shammel Baker, Manuel J. Manard, Jennifer Giddenn and Michael T. Bowers ${ }^{\mathrm{a} *}$ Department of Chemistry, University of California, Santa Barbara, CA 93106

\author{
Supplementary Information
}

\section{Experimental Details}

Desalted dCG was purchased from Sigma-Genosys (The Woodlands, TX) and used without further purification. $\mathrm{M}^{+} \mathrm{dCG}$ complexes were formed in both a home-built MALDI and ESI ion source and the details concerning the experimental setups for the mass spectra and ion mobility measurements have been published previously, so only a brief description will be given [1, 2].

\section{MALDI Details}

2,5-dihydroxybenzoic acid (DHB) was used as the matrix and methanol as the solvent. Approximately $40 \mu \mathrm{L}$ of DHB (100mg/mL), $60 \mu \mathrm{L}$ of dCG (1mg/mL) and $8 \mu \mathrm{L}$ of each metal salt (saturated in methanol) were applied to the sample target and dried. A nitrogen laser ( $\lambda=337 \mathrm{~nm}, 12 \mathrm{~mW}$ power) was used to generate ions in a two-section (Wiley-McLaren) MALDI source. Desorbed ions are then accelerated down a 1-meter flight tube with $9 \mathrm{kV}$ acceleration voltage. The TOF was operated in reflectron mode to obtain high-resolution mass spectra. 
In order to perform ion mobility experiments, the reflectron was turned off and an opposing voltage applied to the ions before the drift cell. This causes deceleration of the ions, which prevents collision-induced dissociation, and allows the ions to be gently injected into a 20 -cm long glass drift cell filled with $\sim 1.5$ torr of helium gas. By controlling the flow rate of warmed or cooled nitrogen through passages surrounding the drift cell, the temperature of the cell can be varied from $80 \mathrm{~K}$ to $500 \mathrm{~K}$ in order to meet the needs of the experiment. A weak, uniform electric field across the cell gently pulls the ions through the He gas at a constant drift velocity. After exiting the drift cell, the ions are gently accelerated through a quadrupole mass filter, mass selecting the ion of interest, and detected with an electron multiplier. The quadrupole is set to a specific mass-tocharge ratio $(\mathrm{m} / \mathrm{z})$ to eliminate any ions that might arise from fragmentation in the drift cell and interfere with the ion mobility experiments. The pulsed source extraction voltage triggers a timing sequence so that the ions are detected as a function of time, yielding an arrival time distribution or ATD. The reduced mobility, $\mathrm{K}_{\mathrm{o}}$, of the ion is accurately determined from a series of ATDs measured at different electric field strengths $(7.5-16 \mathrm{~V} / \mathrm{cm})$ across the drift cell. Through the use of kinetic theory the ion's collision cross-section can also be calculated.

\section{ESI Details}

dCG was resuspended at a concentration of $300 \mu \mathrm{M}$ in $\mathrm{H}_{2} \mathrm{O}$. The solution was then annealed at $70^{\circ} \mathrm{C}$ for 10 minutes, slowly cooled to room temperature, and stored overnight at $10^{\circ} \mathrm{C}$. Approximately $2 \mu \mathrm{L}$ of the dCG solution, $2 \mu \mathrm{L}$ of $\mathrm{H} 2 \mathrm{O}$ and $1 \mu \mathrm{L}$ of each metal salt (saturated in methanol and water) were placed in a metalized glass needle 
(spray tip). Ions were formed by nano-ESI and a continuous beam was injected into an ion funnel. The ion funnel acts as an interface between the source and high vacuum chamber and compresses the stream of ions leaving the capillary. This moves the ions to the drift cell without the aid of high acceleration fields, avoiding high energy ion-neutral collisions. After exiting the ion funnel, the ions are gently injected into a 4-cm long drift cell filled with $\sim 5$ torr of helium gas. A weak, uniform electric field across the cell gently pulls the ions through the He gas at a constant drift velocity. After exiting the drift cell, the ions are gently accelerated through a quadrupole mass filter, which is set to select the mass range of interest to acquire a mass spectrum.

In the ion mobility experiments, a pulsed beam of ions is needed and can be acquired using the ion funnel. By raising the potential of the last lenses in the funnel, the ions become trapped in the ion funnel. Periodically, the potential is lowered for $10 \mu \mathrm{s}$, and a pulse of ions is released. The injection energy of the ions into the drift cell can be varied from 0 to $150 \mathrm{eV}$. At low injection energies, the ions are gently pulsed into the cell and only need a few "cooling" collisions to reach thermal equilibrium with the buffer gas. However, at high injection energies, the larger collision energy leads to internal excitation of the ions before cooling and equilibrium occurs. This transient internal excitation can lead to complex isomerization to the most stable conformers or dissociation of dimers and higher-order oligomers if they are present.

Once the pulsed ions enter the cell, they are quickly thermalized by collisions with $\sim 5$ torr helium buffer gas and are gently pulled through the drift cell by the weak electric field. After exiting the drift cell, the ions enter the quadrupole which is set to a specific mass-to-charge ratio $(\mathrm{m} / \mathrm{z})$ to eliminate any ions that might arise from 
fragmentation in the drift cell and interfere with the ion mobility experiments. The pulsed voltage of the ion funnel triggers a timing sequence, so that the ions are detected as a function of time, yielding an ATD. Similar to the MALDI experiments, the reduced mobility, $K_{o}$, of the ion is accurately determined from a series of ATDs measured at different electric field strengths $(10-23 \mathrm{~V} / \mathrm{cm})$ across the drift cell and through the use of kinetic theory the ion's collision cross-section can also be calculated.

\section{Data Analysis}

The reduced mobility of the mass-selected ions can be obtained from the ATD using Equation 1 [3]

$$
K_{o}=\left(l^{2} \cdot \frac{273}{760 T} \cdot \frac{p}{V} \cdot \frac{1}{t_{A}-t_{o}}\right)
$$

where $l$ is the length of the cell, $T$ is the temperature in Kelvin, $p$ is the pressure of the $\mathrm{He}$ gas (in torr), $V$ is the potential, $t_{A}$ is the ions' arrival time taken from the center of the ATD peak and $t_{o}$ is the amount of time the ion spends outside the drift cell before

reaching the detector. A series of arrival times $\left(t_{A}\right)$ are measured by changing the voltage $(V)$ applied to the drift cell. A plot of $t_{A}$ vs. $p / V$ yields a straight line with a slope inversely proportional to $K_{o}$ and an intercept of $t_{o}$. Once $K_{o}$ is found, the ion's collision cross-section can be obtained using Equation 2

$$
\Omega^{(1,1)}=\frac{3 e}{16 N_{o}}\left(\frac{2 \pi}{\mu k_{b} T}\right)^{1 / 2} \frac{1}{K_{o}}
$$


where $e$ is the charge of the ion, $N_{o}$ is the number density of He at STP, $T$ is temperature, $k_{b}$ is Boltzmann's constant and $\mu$ is the ion-He reduced mass [3].

\section{References}

1. E. S. Baker, J. Gidden, D. P. Fee, P. R. Kemper, S. E. Anderson, and M. T. Bowers Int. J. Mass Spectrom. 227 (2003), p. 205.

2. T. Wyttenbach, P.R. Kemper and M.T. Bowers, Int. J. Mass Spectrom. 212 (2001), p. 13.

3. E.A. Mason and E.W. McDaniel, Transport Properties of Ions in Gases, Wiley, New York (1988). 